\title{
Inhibition of human cervical cancer cell invasion by IL-37 involving runt related transcription factor 2 suppression
}

\author{
Ping Ouyang ${ }^{1 \#}$, Kun Wu ${ }^{1,2 \#}$, Liudan Su${ }^{1,2 \#}$, Weifang $\mathrm{An}^{2}$, Yanhong Bie ${ }^{2}$, He Zhang ${ }^{3}$, Haixian Kang ${ }^{2}$, \\ Enping Jiang ${ }^{1}$, Wei Zhu ${ }^{1}$, Yunhong Yao ${ }^{1}$, Xinrong Hu ${ }^{1}$, Zhangquan Chen ${ }^{1}$, Sen Wang ${ }^{1,2}$ \\ ${ }^{1}$ Guangdong Provincial Key Laboratory of Medical Molecular Diagnostics, Dongguan Scientific Research Center, Guangdong Medical University, \\ Dongguan 523808, China; ${ }^{2}$ Department of Histology and Embryology, Basic Medical College, Guangdong Medical University, Dongguan 523808, \\ China; ${ }^{3}$ Department of Preventive Medicine, School of Public Health, Guangdong Medical University, Dongguan 523808, China \\ Contributions: (I) Conception and design: S Wang; (II) Administrative support: All authors; (III) Provision of study materials or patients: S Wang, Y \\ Bie, Z Chen; (IV) Collection and assembly of data: P Ouyang, K Wu, L Su, W An; (V) Data analysis and interpretation: All authors; (VI) Manuscript \\ writing: All authors; (VII) Final approval of manuscript: All authors. \\ \#These authors contributed equally to this work. \\ Correspondence to: Sen Wang. Guangdong Provincial Key Laboratory of Medical Molecular Diagnostics, Basic Medical College, Guangdong Medical \\ University, \#1 Xincheng Road, Songshan Lake Science and Technology Industry Park, Dongguan 523808, China. \\ Email: wangsen@gdmu.edu.cn; wangsenmol@163.com.
}

Background: IL-37 is a newly anti-inflammatory cytokine whose function is largely unknown in cancer. Our preliminary experiment found IL-37 could inhibit the invasion of human cervical cancer (CC) cells and influence the expression of RUNX family whose function was also unclear in CC. The present study aims to further investigate the effects of IL-37 on cell invasion and runt related transcription factor 2 (RUNX2) expression in CC cell lines.

Methods: Firstly, plasmid overexpressing IL-37 or RUNX2 was transfected into Siha and C33A cells by Hilymax. Then, the effects of IL-37 on the mRNA expression of RUNX1, RUNX2 and RUNX3 gene were detected by quantitative real-time polymerase chain reaction. Protein expression was measured by Western blot and the grayscale scanning analysis. Finally, the effects of IL-37 or RUNX2 on cell invasion were tested by transwell assay.

Results: IL-37 inhibited the mRNA expression of RUNX1 and RUNX2, and increased that of RUNX3 in CC cells. Among the three RUNX genes, RUNX2 showed the most significant change in mRNA expression (decreased by $78.5 \%$ in Siha cells and by $61.5 \%$ in C33A cells) and thus was chosen for the following study. Overexpressed IL-37 inhibited cell invasion by 36.23\% in Siha cells $(\mathrm{P}<0.05)$ and $26.21 \%$ in C33A cells $(\mathrm{P}<0.01)$. Overexpression of RUNX2 promoted cell invasion. Up-regulation of IL-37 suppressed markedly the mRNA and protein expression of RUNX2. Furthermore, overexpressed RUNX2 partially restored the inhibited cell invasion by IL-37 to $86.62 \%$ in Siha cells $(\mathrm{P}<0.01)$ and $87.08 \%$ in C33A cells $(\mathrm{P}<0.01)$.

Conclusions: IL-37 can significantly inhibit the cell invasion of Siha and C33A cells, which involves the suppression of RUNX2.

Keywords: IL-37; runt related transcription factor 2 (RUNX2); cervical cancer (CC); cell invasion; cytokine; tumor

Submitted Jun 20, 2019. Accepted for publication Aug 26, 2019.

doi: 10.21037/atm.2019.09.38

View this article at: http://dx.doi.org/10.21037/atm.2019.09.38 


\section{Introduction}

Growing evidence indicates that IL-37, a new inhibitor of both innate and adaptive immunity, plays a role in tumor (1). As the 7th member of the IL-1 family (IL1F7), IL-37 is produced as a precursor and then cleaved into mature form in the cytoplasm. IL-37 has five isoforms, IL37a, b, c, d and e, which encodes 157 to 218 residues. IL-37b is the biggest isoform and has been identified as a functional gene to inhibit inflammation in many studies $(2,3)$. In the present study, IL-37 means IL-37b isoform. IL-37 can be widely expressed in lymph node, thymus, bone marrow, and other tissues (3). Recent studies suggested an important role of IL-37 in tumor, such as fibrosarcoma (4), liver cancer $(5,6)$, renal cancer $(7)$, lung cancer $(8,9)$. Gao et al. found the development of tumor was significantly inhibited by a single injection of Ad IL-37b, and many injections could completely inhibit fibrosarcoma development (4). Zhao et al. reported IL-37 had lower expression in hepatic cancer and was related to the infiltrating density with CD57 + NK cells (6). In non-small lung cancer tissues, the expression of IL-37 was significantly reduced, and the IL-37 expression levels were associated with cancer status, TNM phase, and poor prognosis (9). Jiang et al. identified IL-37 significantly inhibited the renal cancer cell invasion and proliferation, and promoted cell apoptosis (7). In our previous study, IL37 inhibited cervical cancer (CC) cell proliferation, invasion and apoptosis, but molecules involved in these processes were less well understood $(10,11)$. Thus, this study aimed to investigate the effects of IL-37 on CC cell invasion and the relevant genes.

$\mathrm{CC}$ is the fifth leading cause of cancer death in women (12). About 266,000 women die of it every year (13). Understanding the molecular pathways and genes which affect the CC progression will help improve the treatment and reduce the mortality. Runt related transcription factor 2 (RUNX2) is a member of the RUNX family whose function in CC is still not clear. RUNX2 is a key regulator of normal bone development, homeostasis and remodeling (14-16). Further, mounting evidence showed that RUNX2 activated genes associated with tumor genesis and metastasis, and promote tumor cell invasion in cancers, such as breast cancer (17-19), prostate cancer (20,21), thyroid cancers (22), osteosarcoma (23-25), and melanoma (26). Our preliminary study experiment found that IL-37 could inhibit human CC cell invasion and influence the expression of RUNX family. In the present study, we aim to investigate the role of IL-37 and the correlation of IL-37 and RUNX2 in CC cells. To the best of our knowledge, it is the first time to report the correlation of IL-37 and RUNX2 expression in CC. The results will be helpful to understand the biological function of IL-37 and to provide new potential molecule for CC therapy.

\section{Methods}

\section{Cell lines and transfection}

Human CC cell lines, Siha and C33A were purchased from the China Center for Type Culture Collection (CCTCC) (Wuhan, China) as previously described $(10,26)$. The cells were maintained in DMEM medium containing $10 \%$ fetal bovine serum (FBS) purchased from Invitrogen, supplementary with $2.0 \mathrm{mmol} / \mathrm{L} \mathrm{L}$-glutamine, $50 \mathrm{U}$ penicillin and $50 \mu \mathrm{g} / \mathrm{mL}$ streptomycin. All cells were routinely cultured in a $5 \% \mathrm{CO}_{2}$ atmosphere at $37^{\circ} \mathrm{C}$. The empty vector pIRES2-EGFP was purchased from Shanghai Geneary Biotech Co., Ltd. (Shanghai, China). The expression plasmid pIRES2-EGFP/ IL-37 which expressed IL-37 (NM_014439) in eukaryotic cells had been described in our previous study (10). The expression plasmid pGV230-RUNX2 which expressed RUNX2 (NM_001015051) in eukaryotic cells was cloned into XhoI/KpnI clone sites and sequenced by Genechem Co., Ltd, China.

For transfection, cells were plated onto 6-well plates (Corning, USA) at a density of $2.5 \times 10^{5}$ cells per well. Cells were transfected with Hilymax (Dojindo, Shanghai, China) reagents in serum- and antibiotic-free OptiMEM (GibcoBRL, USA) according to the manufacturer's instructions. The ratio of Hilymax $(\mu \mathrm{L})$ to DNA $(\mu \mathrm{g})$ was 15:3.

\section{Reverse transcription-quantitative polymerase chain reaction (RT-qPCR) analysis}

RT-qPCR was performed for detecting the mRNA levels of IL-37, GAPDH, RUNX1, RUNX2, and RUNX3. Total RNAs were extracted from the cells using TRIzol (Invitrogen, Carlsbad, CA, USA) according to the manufacturer's instructions. QPCR was performed on Prism 7500 (ABI, Foster City, CA, USA) by using standard SYBR green assay protocol. Primers were as follows: IL-37-F, 5'-GATCACAAAGTACTGGTCCTGG-3'; IL-37-R, 5'-TCCTTTATCCTTGTCACAGTAG-3'. RUNX1-F, 5' - CACCTACCACAGAGCCATCA-3'; RUNX1-R, 
5'- CTCGGAAAAGGACAAGCTCC-3'. RUNX2-F, 5 '-TCTTCACAAATCCTCCCC-3'; RUNX2-R, 5'-TGGATTAAAAGGACTTGGTG-3'. RUNX3-F, 5'TTATGAGGGGTGGTTGTATGTGGG-3'; RUNX3-R, 5' - AAACAACCAACACAAACACCTCC-3'. GAPDH was used as a normalization control, and the primers were 5'-TGACTTCAACAGCGACACCCA-3' (forward) and 5'-CACCCTGTTGCTGTAGCCAAA-3' (reverse). PCR was performed in triplicate in a $20 \mu \mathrm{L}$ reaction volume containing $10 \mu \mathrm{L}$ Platinum ${ }^{\circledR} \mathrm{SYBR}^{\circledR}$ Green (Invitrogen, Guangzhou, China), $0.8 \mu \mathrm{L}$ forward primer $(10 \mu \mathrm{M})$, $0.8 \mu \mathrm{L}$ reverse primer $(10 \mu \mathrm{M}), 1 \mu \mathrm{L}$ cDNA and $7.4 \mu \mathrm{L}$ $\mathrm{ddH}_{2} \mathrm{O}$. The reaction conditions were $95^{\circ} \mathrm{C}$ for $10 \mathrm{~min}$, 40 cycles of $95^{\circ} \mathrm{C}$ for $15 \mathrm{~s}$ and $60^{\circ} \mathrm{C}$ for $1 \mathrm{~min}$. Data were analyzed using the sequence detection software (version 1.6.3) supplied by Applied Biosystems (ABI). Each experiment was performed in triplicate. The relative mRNA levels were calculated and normalized using the $2-{ }^{\Delta \Delta C t}$ method relative to GAPDH (27).

\section{Western blotting}

Cells were lysed in RIPA buffer (Beyotime, Shanghai, China) and the lysates were collected into tubes and centrifuged at $12,000 \mathrm{~g}$ for $20 \mathrm{~min}$ at $4{ }^{\circ} \mathrm{C}$. The supernatants were collected and protein concentration was quantified with BCA Protein Quantitative Kit (Beyotime, Shanghai, China). Then, western blotting was performed according to the manuals. Equal amount of protein $(200 \mu \mathrm{g})$ was separated on $10 \%$ SDS-PAGE and transferred to PVDF membranes (Millipore, Billerica, MA, USA). The membrane were then blocked with $5 \%$ non-fat milk for $1 \mathrm{~h}$ and incubated overnight at $4{ }^{\circ} \mathrm{C}$ with rabbit antihuman antibodies against IL-37 (Abcam, Cambridge, MA, USA, ab116282; diluted 1:1,000), RUNX2 (Abcam, Cambridge, MA, USA, ab23981; diluted 1:1,000), and GAPDH (Goodhere Technology, Hangzhou, China; ABP-R001; diluted 1:500), respectively. In the next day, the membrane was-incubated with HRP-conjugated secondary antibody (Beyotime, Shanghai, China; diluted 1:1,000) for $1 \mathrm{~h}$ and detected by enhanced chemiluminescence (ECL). Reactive bands were revealed with Immobilon Western Chemiluminescent kit (WBKLS0100, Millipore, USA) by Cobas e601 automatic chemiluminescence image analysis system (Roche, USA). Each experiment was performed in triplicate.

\section{Cell invasion assay}

Cell invasion ability was detected by transwell assay as described previously (10). The upper chambers were precoated with Matrigel (BD Bioscience, San Jose, CA, USA). Cells $\left(6.0 \times 10^{4}\right.$ cells $/$ well $)$ were placed into the upper chambers (BD Bioscience, San Jose, CA, USA) containing $10 \%$ FBS. The lower chambers were filled with RPMI- 1640 with $20 \%$ FBS. After incubation for $48 \mathrm{~h}$ for Siha or C33A cells at $37^{\circ} \mathrm{C}$, cells on the lower surface of the membranes were fixed and stained with $0.1 \%$ crystal violet, and five random fields of vision were selected to be counted under a light microscope (Olympus, 200×). Each experiment was performed in triplicate.

\section{Statistical analysis}

Statistical analyses were performed using SPSS 15.0 (http:// spss.en.softonic.com/). Independent sample $t$-test was used for analyzing two groups and one-way analysis of variance (ANOVA) was used for examining multiple groups. Rates were compared using the Chi-square test. The level of statistical significance was set at $\mathrm{P}<0.05$ or $\mathrm{P}<0.01$.

\section{Results}

\section{IL-37 overexpression inbibited cell invasion in both of the two CC cell lines}

The protein encoded by IL-37b contains 218 amino acids with the molecular weight of $\sim 24 \mathrm{kDa}$. Protein bands of IL-37 in normal Siha and C33A cells were hardly detected. Plasmid pIRES2-EGFP/IL-37 was transfected into CC cells to overexpress IL-37 (Siha-IL-37 group and C33A-IL-37 group, respectively). In Siha cells, compared with that of the control group (Siha-NC), the mRNA level of IL-37 in Siha-IL-37 group was 5,691.67-fold $(\mathrm{P}<0.001$, Figure $1 A)$. The results of Western Blotting showed the protein expression level of IL-37 in Siha-IL-37 group achieved 7.47 -fold $(\mathrm{P}<0.01$, Figure $1 B)$. Compared with that of the Siha-NC group, the cell invasion ability of Siha-IL-37 group reduced significantly, and the invasion rate was downregulated by $36.23 \%(\mathrm{P}<0.05$, Figure $1 C)$. While in $\mathrm{C} 33 \mathrm{~A}$ cells, compared with that of the control group (C33A$\mathrm{NC}$ ), the mRNA level of C33A-IL-37 group obtained 2425.67-fold $(\mathrm{P}<0.01$, Figure 1D). The results of Western Blotting showed the protein expression of IL-37 increased 


\section{A}

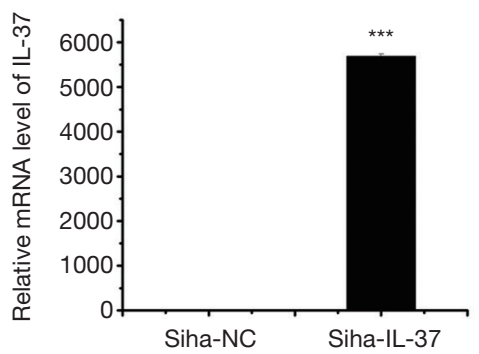

C

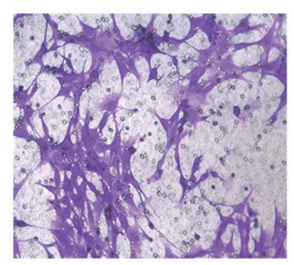

Siha-NC

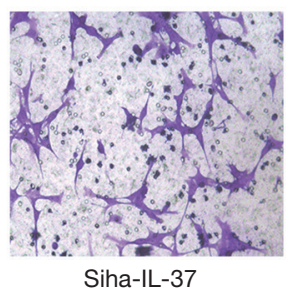

D

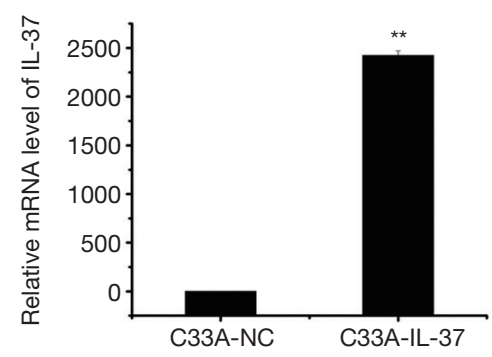

F
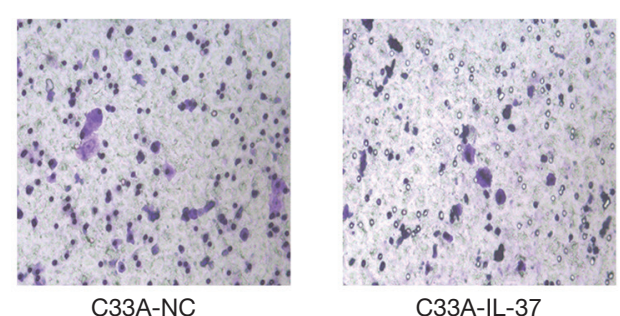

B
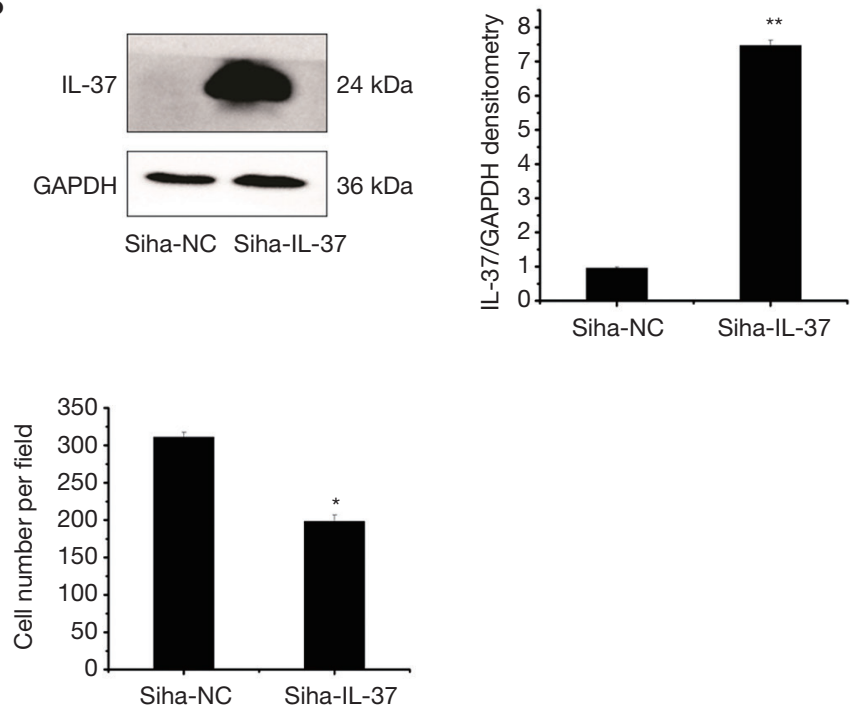

E
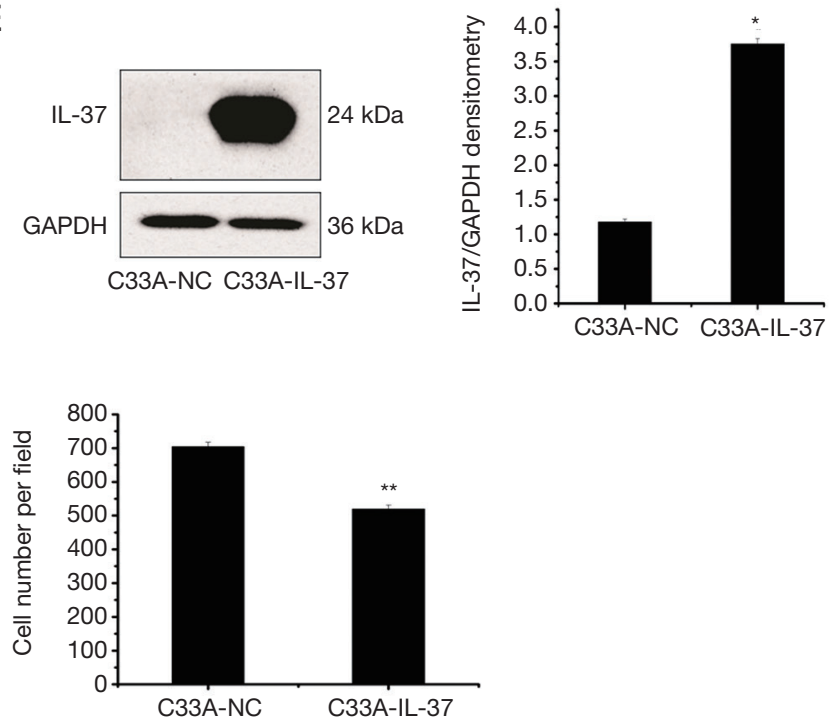

Figure $1 \mathrm{IL}-37$ inhibited cell invasion of Siha and C33A cells. Expression of IL-37 was up-regulated by $I L-37$ gene transfection in Siha and C33A cells. (A) The relative mRNA level and (B) protein level of IL-37 in Siha-NC (transfecting pIRES2-EGFP empty vector) and SihaIL-37 groups (transfecting pIRES2-EGFP/IL-37); (C) representative photomicrographs of invaded Siha cells in Siha-NC and Siha-IL-37 groups, and the quantified numbers in the two groups; (D) the relative mRNA level and (E) protein level of IL-37 in C33A-NC (transfecting pIRES2-EGFP empty vector) and C33A-IL-37 (transfecting pIRES2-EGFP/IL-37) groups; (F) representative photomicrographs of invaded C33A cells in C33A-NC and C33A-IL-37 groups, and the quantified numbers in the two groups. Crystal violet staining (20x). *, $\mathrm{P}<0.05$; **, $\mathrm{P}<0.01 ;$ ***, $\mathrm{P}<0.001$.

about 2.18-fold $(\mathrm{P}<0.05$, Figure $1 E)$. Compared with that of the C33A-NC group, the cell invasion ability of C33AIL-37 group reduced significantly, and the invasion rate was down-regulated by $26.21 \%(\mathrm{P}<0.01$, Figure $1 F)$. These results suggested IL-37 inhibited cell invasion in both Siha and C33A cells.

\section{IL-37 inbibited the expression of RUNX2 in both of the two CC cell lines}

In our preliminary study, we found in Siha and C33A cells, the expression of RUNX family members changed in varying degrees. IL-37 inhibited the mRNA expression of RUNX1 and RUNX2, and increased the mRNA expression 
A

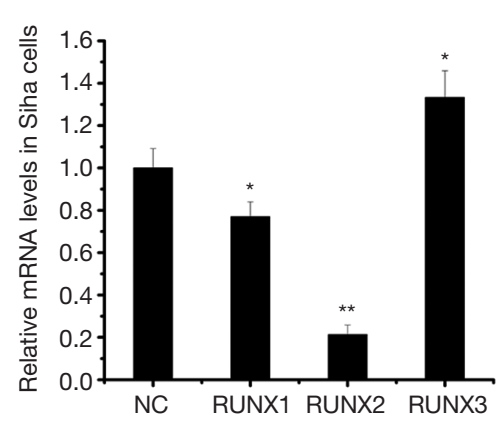

C

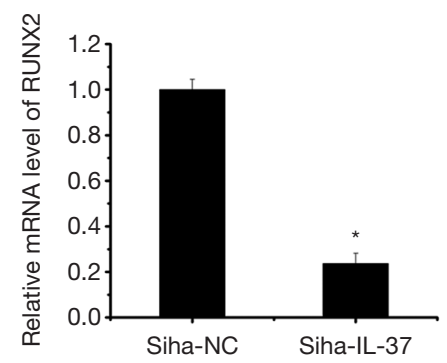

$\mathrm{E}$

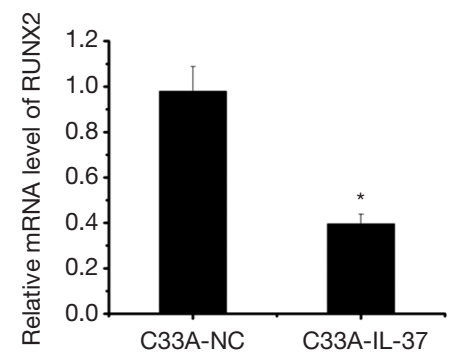

B

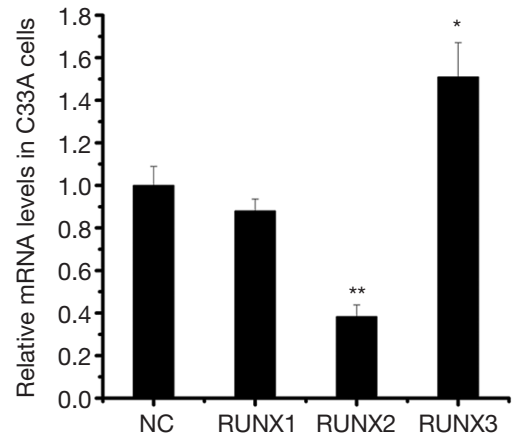

D
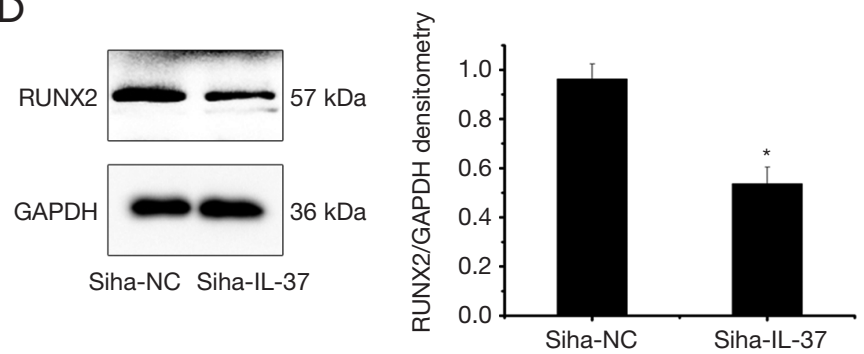

$\mathrm{F}$
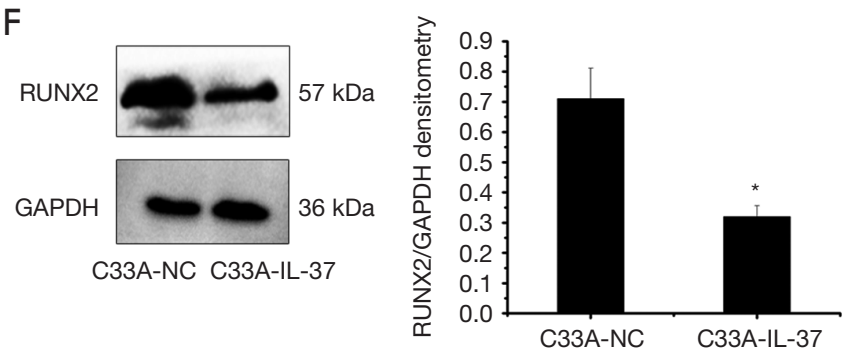

Figure 2 IL-37 suppressed RUNX2 expression in both cells. Up-regulation of IL-37 influenced the mRNA expression of RUNX family members. (A) the relative mRNA levels of RUNX1, RUNX2 and RUNX3 in Siha-NC (transfecting pIRES2-EGFP empty vector) and Siha-IL-37 (transfecting pIRES2-EGFP/IL-37) groups; and (B) in C33A-NC (transfecting pIRES2-EGFP empty vector) and C33A-IL-37 (transfecting pIRES2-EGFP/IL-37) groups; (C) the relative mRNA level and (D) protein level of RUNX2 in Siha-NC and Siha-IL-37 groups; (E) the relative mRNA level and (F) protein level of RUNX2 in C33A-NC and C33A-IL-37 groups. *, $\mathrm{P}<0.05$; **, $\mathrm{P}<0.01$. RUNX2, runt related transcription factor 2 .

of RUNX3 (Figure 2A,B), among which, RUNX2 showed the most significant change in mRNA expression (decreased by $78.5 \%$ in Siha cells and by $61.5 \%$ in C33A cells). Thus, the experiments were performed using RUNX2 gene in next study.

To identify the relationship between IL-37 and RUNX2, we detected the mRNA and protein expression levels of RUNX2 after transfecting pIRES2-EGFP/IL-37 in Siha and C33A cells. RUNX2 encodes a protein of 521 amino acids with the molecular weight of $\sim 56.6 \mathrm{Kda}$ (28). Compared with that of the Siha-NC group, the mRNA of RUNX2 in Siha-IL-37 reduced by $76.10 \%(\mathrm{P}<0.05$, Figure $2 C$ ), and the protein level reduced by $43.75 \%$
$(\mathrm{P}<0.05$, Figure 2D). While in C33A cells, compared with that of the C33A-NC group, the mRNA reduced by $59.08 \%(\mathrm{P}<0.05$, Figure $2 E)$, and the protein reduced by $54.93 \%(\mathrm{P}<0.05$, Figure $2 F)$, respectively.

\section{RUNX2 overexpression promoted cell invasion}

To determine the role of RUNX2 in cell invasion, RUNX2 was overexpressed in cancer cells. In Siha cells transfected with RUNX2, compared with that of the Siha-NC group, the mRNA expression level of RUNX2 was 2.57 -fold $(\mathrm{P}<0.05$, Figure $3 A)$ and the protein of RUNX2 increased about 3.00-fold $(\mathrm{P}<0.01$, Figure 3B). Compared with that of 
Page 6 of 10

A

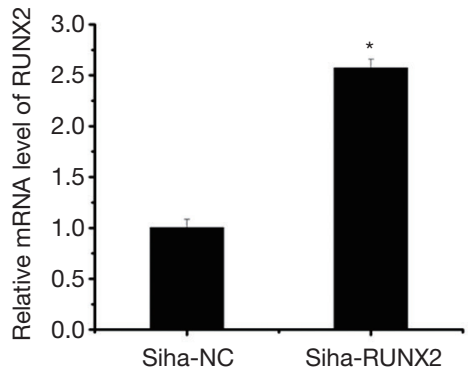

C

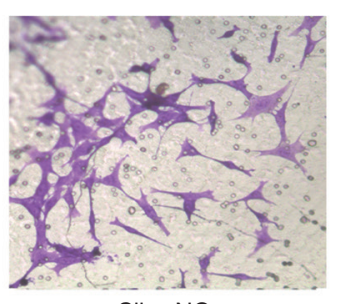

Siha-NC

D

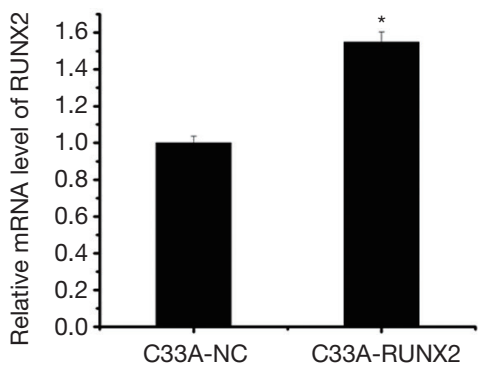

F

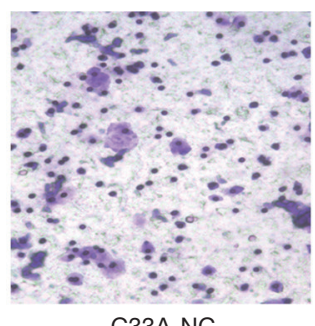

B
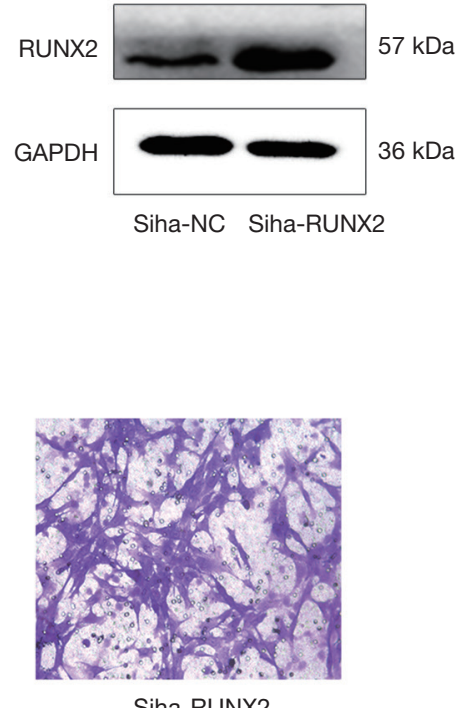

Siha-RUNX2

E
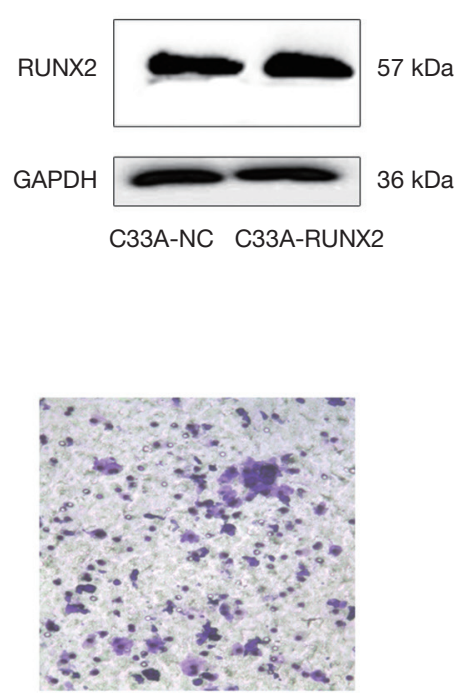

C33A-RUNX2
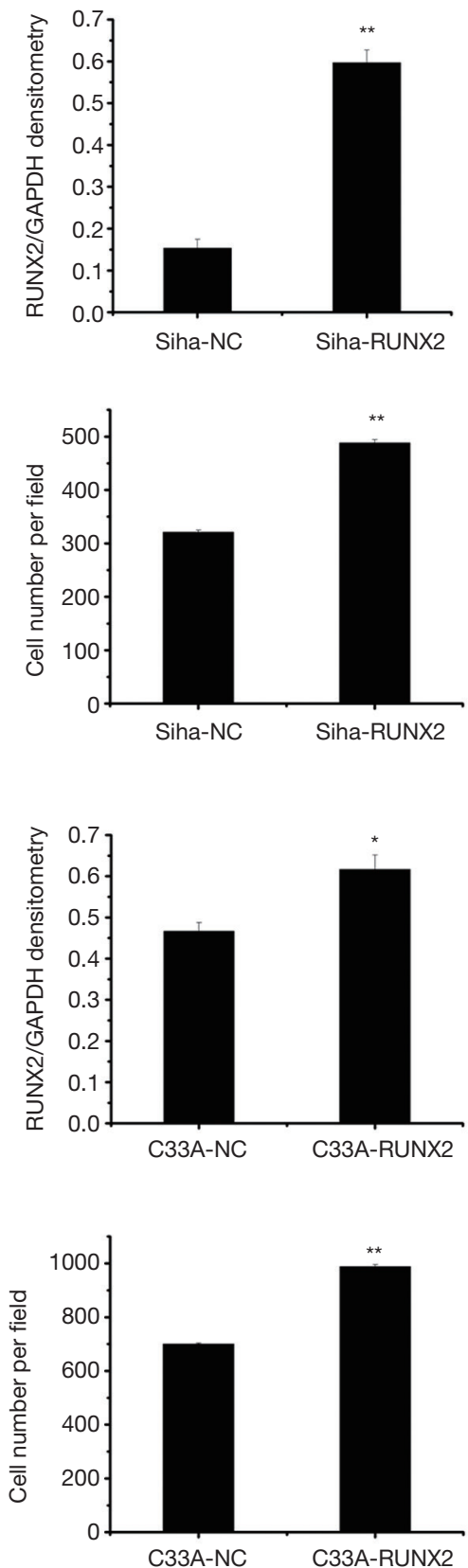

Figure 3 RUNX2 promoted cell invasion in both cells. Plasmid overexpressing RUNX2 was transfected into Siha and C33Acells. (A) The relative mRNA level and (B) protein level of RUNX2 in Siha-NC (transfecting pGV230 empty vector) and Siha-RUNX2 groups (transfecting pGV230-RUNX2); (C) representative photomicrographs of invaded Siha cells in Siha-NC and Siha-RUNX2 groups, and the quantified numbers in the two groups; (D) the relative mRNA level and (E) protein level of RUNX2 in C33A-NC (transfecting pGV230 empty vector) and C33A-RUNX2 (transfecting pGV230-RUNX2) groups; (F) representative photomicrographs of invaded C33A cells in C33A-NC and C33A-RUNX2 groups, and the quantified numbers in the two groups. Crystal violet staining $(20 \times)$. *, $\mathrm{P}<0.05$; **, P<0.01. RUNX2, runt related transcription factor 2 . 
A

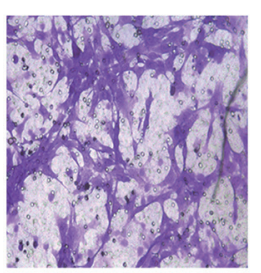

Siha-NC

C

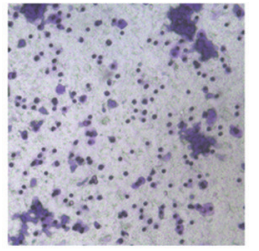

C33A-NC

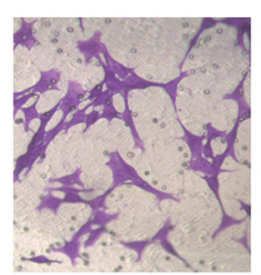

Siha-IL-37

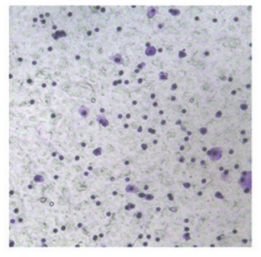

C33A-IL37

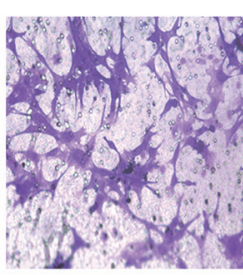

Siha-IL-37+RUNX2

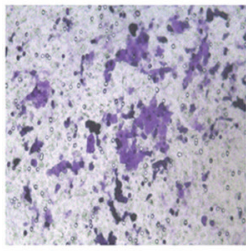

C33A-IL-37+RUNX2
B

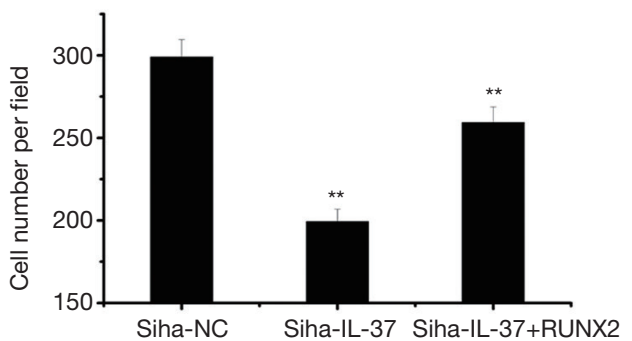

D

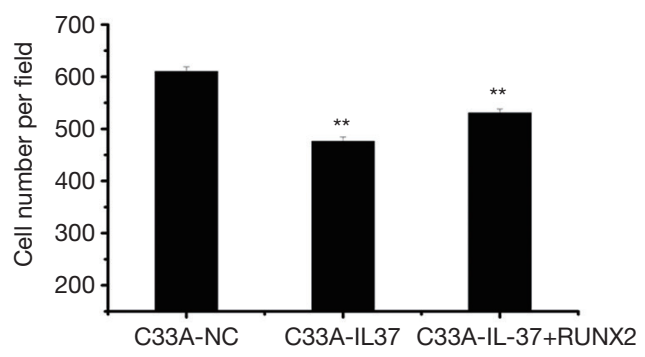

Figure 4 RUNX2 partially restored the inhibited cell invasion ability by IL-37, crystal violet staining (20x). IL-37 was firstly up-regulated in CC cells by gene transfection. Then, RUNX2 plasmid was transfected into cells. (A) Representative photomicrographs of invaded Siha cells transfected with pIRES2-EGFP + pGV230 empty vectors, pIRES2-EGFP/IL-37 and pIRES2-EGFP/IL-37 + pGV230-RUNX2, respectively; and (B) quantified numbers of invaded Siha cells in the three groups; (C) representative photomicrographs of invaded C33A cells transfected with pGV230 + pIRES2-EGFP empty vectors, pIRES2-EGFP/IL-37 and pIRES2-EGFP/IL-37 + pGV230-RUNX2, respectively; and (D) quantified numbers of invaded C33A cells in the three groups. ${ }^{* *}, \mathrm{P}<0.01$. RUNX2, runt related transcription factor 2.

the Siha-NC group, the cell invasion ability of Siha-RUNX2 group increased significantly, and the invasion rate was 1.52fold $(\mathrm{P}<0.01$, Figure $3 C)$. While in C33A cells, the mRNA of RUNX2 increased by $55.00 \%(\mathrm{P}<0.05$, Figure $3 D)$, and the protein level increased by $32.13 \%(\mathrm{P}<0.05$, Figure 3E), respectively. Compared with that of the C33A$\mathrm{NC}$ group, the cell invasion ability of C33A-RUNX2 group increased significantly, and the invasion rate was 1.41 fold $(\mathrm{P}<0.01$, Figure $3 F)$. These results suggested RUNX2 promoted cell invasion in both Siha and C33A cells.

\section{Up-regulation of RUNX2 partially restored the cell invasion ability down-regulated by IL-37}

In Siha cells, the cell invasion ability of Siha-IL-37 group was $66.56 \%$ of that of the Siha-NC group (Figure $4 A$ ). Then, up-regulation of RUNX2 by gene transfection restored the cell invasion rate to $86.62 \%$ (Figure $4 B$ ). Similar results were shown in $\mathrm{C} 33 \mathrm{~A}$ cells. The cell invasion ability of C33A-IL-37 group was $78.01 \%$ of that of the C33A-NC group (Figure $4 C$ ). Then, up-regulation of RUNX2 partially restored the cell invasion rate to $87.08 \%$ (Figure $4 D$ ). These results suggested RUNX2 could partially restore the cell invasion ability of Siha and C33A cells, and RUNX2 took part in the invasion inhibition by IL-37.

\section{Discussion}

In this study, the effect of IL-37 on cell invasion was investigated. We found IL-37 played an inhibitory role in CC cell invasion, which suggested IL-37 might work as an anti-tumor molecule in Siha and C33A cells. This result was consistent with many previous studies. In fibrosarcoma, IL-37 showed significant tumor suppression in mice (4). In renal cell carcinoma, IL-37 expression was observed to inhibit cancer cell proliferation and invasion through IL-6/ Stat3 pathway (7). In colon cancer cells, overexpression of IL-37 inhibited cell migration and invasion by suppressing $\beta$-catenin (29). These studies implicated the inhibition ability of IL-37 on tumor cells.

Additionally, IL-37 also had inhibition ability on tumor microenvironment. In hepatocellular carcinoma, IL37 expression was decreased, but increased intratumoral expression of IL-37 proportionally positively correlated with the overall survival and disease-free survival (6). The mechanism of the antitumor effect of IL-37 is activating 
immunity in the tumor microenvironment through the regulation of NK cells. In breast cancer, IL-37 was upregulated and showed the antitumor effects by influencing the tumor microenvironment (30). In non-small lung cancer, Ge et al. found IL-37 expression was up-regulated in non-tumor tissues but down-regulated in lung cancer tissues, they further suggested IL-37 repression of the development of lung cancer may be through tumor angiogenesis inhibition (9). However, in ovarian cancer, the authors found high serum IL-37 levels were associated with an unfavorable prognosis of human epithelial ovarian cancer (EOC) patients (31). More data is needed and the relationship between IL-37 and CC microenvironments may be a good direction in future studies.

Furthermore, RUNX2 could promote cell invasion in CC cells. Guo et al. found that RUNX2 significantly increased the migration and invasion ability of gastric cancer (GC) cells and enhanced the invasion and metastatic potential in an orthotopic GC model of nude mice (32). In osteosarcoma, Zeng et al. found down-regulating RUNX2 by siRNA could inhibit the invasion of osteosarcoma SAOS2 cells (25), and Xie et al. found RUNX2 promoted cell invasion of 143B osteosarcoma cells (24). Zhao et al. found up-regulation of RUNX2 resulted in a greater cell invasive capacity in bladder cancer (33). In addition, RUNX2 was able to promote cell invasion in human colorectal cancer (34), breast cancer (18), ovarian cancer (35), prostate cancer (21), lung cancer $(36,37)$, and human glioma (38). However, to our knowledge, the function of RUNX2 in CC was unclear.

RUNX2 was involved in the inhibition of cell invasion by IL-37, no similar report was found before. IL-37 gene was transfected into cancer cells and decreased the expression of mRNA and protein of RUNX2, and RUNX2 overexpression can rescue the cell invasion reduced by IL-37 in CC cells. All these results indicated RUNX2 was involved in the anti-tumor processes of IL-37 induced. Mature IL-37 can translocate into the nucleus and activate many transcriptional factors. Our previous study had proved IL-37 could regulate STAT3 signaling in CC cells HeLa and C33A. Here, we also showed the participation of RUNX2 in the inhibited cell invasion by IL-37. To our knowledge, the interaction between the two proteins was reported for the first time. This result helps to further understand the molecular network associated with IL-37 in CC.

In addition, due to more and more stringent ethical review, we could not collect enough human biopsies to detect the expression of those genes. Thus, the present version of this study focused on the cell invasion in cancer cell lines in vitro and the results did show that RUNX2 promoted cancer cell invasion and was involved in the cell invasion inhibition by IL-37 in vitro. The preliminary results showed that IL-37 up regulated RUNX3 which might be a tumor suppressor for CC (39), suggesting that RUNX3 up regulation might also be related to the function of IL-37. However, RUNX3 was not the mostly changed gene among the RUNX family and was not the main clue for this study, we hope to further research it in next study.

In the present study, C33a cells are HPV-negative and $\mathrm{SiHa}$ cells are HPV16-positive which is the most important HPV subtype in HPV infection. The inhibitory ability of IL-37 on SiHa cells seemed a little weaker than that on C33a cells. The data in the HPV-negative and HPVpositive cell lines was different, the difference was not so significant here. HPV16 E6 gene is one of the main oncogene for cervical carcinogenesis which is mainly related to P53 pathway. Two studies speculated that IL-37 might regulate P53 $(40,41)$ which mediated HPV E6-induced carcinogenesis. Thus, IL-37 might play a role in HPVinduced pathological process by regulating $\mathrm{P} 53$, which might be a potential idea for the next study.

\section{Conclusions}

In conclusion, the present study demonstrates that IL-37 can significantly inhibit cell invasion in CC cells Siha and C33A. RUNX2 has an opposite effect. The anti-tumor effect of IL-37 is, at least in part, via the suppression of RUNX2 in CC cells.

\section{Acknowledgments}

Funding: This study is supported by the Natural Science Foundation of Guangdong Province, China (2018A030307026), by Science and Technology Planning Project of Guangdong Province, China (2016A020215147), by Medical Science and Technology Research Fund of Guangdong province, China (A2017605, A2016208), by Students' innovative experiment program of Guangdong medical university (2016ZYDS001, 2016ZYDS004, 2016ZYDS005, 2017ZZDS005), by student's platform for innovation and entrepreneurship training program (GDMU2016006, GDMU2016024, GDMU2016037, GDMU2017004, 201710571006, 201710571024, 201710571037), by National Natural Science Foundation of China (81502899, 81302244, 81200082). 


\section{Footnote}

Conflicts of Interest: The authors have no conflicts of interest to declare.

Ethical Statement: This study didn't include any human tissues or animal experiments; thus, no ethics approval was needed. The authors are accountable for all aspects of the work in ensuring that questions related to the accuracy or integrity of any part of the work are appropriately investigated and resolved.

\section{References}

1. Ding VA, Zhu Z, Xiao H, et al. The role of IL-37 in cancer. Med Oncol 2016;33:68.

2. Dinarello CA, Bufler P. Interleukin-37. Semin Immunol 2013;25:466-8.

3. Boraschi D, Lucchesi D, Hainzl S, et al. IL-37: a new antiinflammatory cytokine of the IL-1 family. Eur Cytokine Netw 2011;22:127-47.

4. Gao W, Kumar S, Lotze MT, et al. Innate immunity mediated by the cytokine IL-1 homologue 4 (IL-1H4/ IL-1F7) induces IL-12-dependent adaptive and profound antitumor immunity. J Immunol 2003;170:107-13.

5. Liu R, Tang C, Shen A, et al. IL-37 suppresses hepatocellular carcinoma growth by converting $\mathrm{pSmad} 3$ signaling from $\mathrm{JNK} / \mathrm{pSmad} 3 \mathrm{~L} / \mathrm{c}-\mathrm{Myc}$ oncogenic signaling to $\mathrm{pSmad} 3 \mathrm{C} / \mathrm{P} 21$ tumor-suppressive signaling. Oncotarget 2016;7:85079-96.

6. Zhao JJ, Pan QZ, Pan K, et al. Interleukin-37 mediates the antitumor activity in hepatocellular carcinoma: role for CD57+ NK cells. Sci Rep 2014;4:5177.

7. Jiang Y, Wang Y, Liang L, et al. IL-37 mediates the antitumor activity in renal cell carcinoma. Med Oncol 2015;32:250.

8. Chen YH, Zhou BY, Wu GC, et al. Effects of exogenous IL-37 on the biological characteristics of human lung adenocarcinoma A549 cells and the chemotaxis of regulatory T cells. Cancer Biomark 2018;21:661-73.

9. Ge G, Wang A, Yang J, et al. Interleukin-37 suppresses tumor growth through inhibition of angiogenesis in nonsmall cell lung cancer. J Exp Clin Cancer Res 2016;35:13.

10. Wang S, An W, Yao Y, et al. Interleukin 37 Expression Inhibits STAT3 to Suppress the Proliferation and Invasion of Human Cervical Cancer Cells. J Cancer 2015;6:962-9.

11. Ouyang P, An W, Chen R, et al. IL-37 promotes cell apoptosis in cervical cancer involving Bim up-regulation.
OncoTargets and Therapy 2019;12:2703-12.

12. Cancer Genome Atlas Research Network, Albert Einstein College of Medicine, Analytical Biological Services, et al. Integrated genomic and molecular characterization of cervical cancer. Nature 2017;543:378-84.

13. Koh W-J, Greer BE, Abu-Rustum NR, et al. Cervical Cancer, Version 2.2015. Journal of the National Comprehensive Cancer Network 2015;13:395-404.

14. Xu W, Chen Q, Liu C, et al. A novel, complex RUNX2 gene mutation causes cleidocranial dysplasia. BMC Med Genet 2017;18:13.

15. Thiagarajan L, Abu-Awwad HAM, Dixon JE. Osteogenic Programming of Human Mesenchymal Stem Cells with Highly Efficient Intracellular Delivery of RUNX2. Stem Cells Transl Med 2017;6:2146-59.

16. Murakami J, Ishii M, Suehiro F, et al. Vascular endothelial growth factor-C induces osteogenic differentiation of human mesenchymal stem cells through the ERK and RUNX2 pathway. Biochem Biophys Res Commun 2017;484:710-8.

17. Tandon M, Othman AH, Ashok V, et al. The role of Runx2 in facilitating autophagy in metastatic breast cancer cells. J Cell Physiol 2018;233:559-71.

18. Vishal M, Swetha R, Thejaswini G, et al. Role of Runx2 in breast cancer-mediated bone metastasis. Int J Biol Macromol 2017;99:608-14.

19. Wysokinski D, Blasiak J, Pawlowska E. Role of RUNX2 in Breast Carcinogenesis. Int J Mol Sci 2015;16:20969-93.

20. Yang Y, Bai Y, He Y, et al. PTEN Loss Promotes Intratumoral Androgen Synthesis and Tumor Microenvironment Remodeling via Aberrant Activation of RUNX2 in Castration-Resistant Prostate Cancer. Clin Cancer Res 2018;24:834-46.

21. Colden M, Dar AA, Saini S, et al. MicroRNA-466 inhibits tumor growth and bone metastasis in prostate cancer by direct regulation of osteogenic transcription factor RUNX2. Cell Death Dis 2017;8:e2572.

22. Sancisi V, Manzotti G, Gugnoni M, et al. RUNX2 expression in thyroid and breast cancer requires the cooperation of three non-redundant enhancers under the control of BRD4 and c-JUN. Nucleic Acids Res 2017;45:11249-67.

23. Liang GH, Liu N, He MT, et al. Transcriptional regulation of Runx2 by HSP90 controls osteosarcoma apoptosis via the AKT/GSK-3beta/beta-catenin signaling. J Cell Biochem 2018;119:948-59.

24. Xie Y, Sun W, Deng Z, et al. MiR-302b Suppresses Osteosarcoma Cell Migration and Invasion by Targeting 
Runx2. Sci Rep 2017;7:13388.

25. Zeng $\mathrm{H}, \mathrm{Xu} \mathrm{X}$. RUNX2 RNA interference inhibits the invasion of osteosarcoma. Oncol Lett 2015;9:2455-8.

26. Boregowda RK, Medina DJ, Markert E, et al. The transcription factor RUNX2 regulates receptor tyrosine kinase expression in melanoma. Oncotarget 2016;7:29689-707.

27. Livak KJ, Schmittgen TD. Analysis of relative gene expression data using real-time quantitative PCR and the 2(-Delta Delta C(T)) Method. Methods 2001;25:402-8.

28. Nagel AK, Ball LE. O-GlcNAc Modification of the runt-Related Transcription Factor 2 (Runx2) Links Osteogenesis and Nutrient Metabolism in Bone Marrow Mesenchymal Stem Cells. Molecular \& Cellular Proteomics 2014;13:3381-95.

29. Yan X, Zhao J, Zhang R. Interleukin-37 mediates the antitumor activity in colon cancer through beta-catenin suppression. Oncotarget 2017;8:49064-75.

30. Wang WQ, Zhao D, Zhou YS, et al. Transfer of the IL$37 \mathrm{~b}$ gene elicits anti-tumor responses in mice bearing 4T1 breast cancer. Acta Pharmacol Sin 2015;36:528-34.

31. Huo J, Hu J, Liu G, et al. Elevated serum interleukin-37 level is a predictive biomarker of poor prognosis in epithelial ovarian cancer patients. Arch Gynecol Obstet 2017;295:459-65.

32. Guo ZJ, Yang L, Qian F, et al. Transcription factor RUNX2 up-regulates chemokine receptor CXCR4 to promote invasive and metastatic potentials of human gastric cancer. Oncotarget 2016;7:20999-1012.

Cite this article as: Ouyang $\mathrm{P}, \mathrm{Wu} \mathrm{K}, \mathrm{Su} \mathrm{L}, \mathrm{An} \mathrm{W}$, Bie $\mathrm{Y}$, Zhang H, Kang H, Jiang E, Zhu W, Yao Y, Hu X, Chen Z, Wang S. Inhibition of human cervical cancer cell invasion by IL-37 involving runt related transcription factor 2 suppression. Ann Transl Med 2019;7(20):568. doi: 10.21037/atm.2019.09.38
33. Zhao X, Ji Z, Xie Y, et al. MicroRNA-154 as a prognostic factor in bladder cancer inhibits cellular malignancy by targeting RSF1 and RUNX2. Oncol Rep 2017;38:2727-34.

34. Wen D, Li S, Jiang W, et al. miR-539 inhibits human colorectal cancer progression by targeting RUNX2. Biomed Pharmacother 2017;95:1314-20.

35. Li N, Wang L, Tan G, et al. MicroRNA-218 inhibits proliferation and invasion in ovarian cancer by targeting Runx2. Oncotarget 2017;8:91530-41.

36. Bai X, Meng L, Sun H, et al. MicroRNA-196b Inhibits Cell Growth and Metastasis of Lung Cancer Cells by Targeting Runx2. Cell Physiol Biochem 2017;43:757-67.

37. Zheng QW, Zhou YL, You QJ, et al. WWOX inhibits the invasion of lung cancer cells by downregulating RUNX2. Cancer Gene Ther 2016;23:433-8.

38. Zhu Y, Zhao H, Feng L, et al. MicroRNA-217 inhibits cell proliferation and invasion by targeting Runx2 in human glioma. Am J Transl Res 2016;8:1482-91.

39. Li Z, Fan P, Deng M, et al. The roles of RUNX3 in cervical cancer cells in vitro. Oncol Lett 2018;15:8729-34.

40. Conti P, Lessiani G, Kritas SK, et al. Mast cells emerge as mediators of atherosclerosis: Special emphasis on IL-37 inhibition. Tissue Cell 2017;49:393-400.

41. Carinci F, Lessiani G, Spinas E, et al. Mast cell and cancer with special emphasis on il-37 an anti-inflammatory and inhibitor of innate immunity: new frontiers. J Biol Regul Homeost Agents 2016;30:945-50. 